\title{
Video Article \\ Introducing Point Mutations into Human Pluripotent Stem Cells using Seamless Genome Editing
}

\author{
Yu Wang ${ }^{1}$, Andrew J. H. Smith ${ }^{1}$, David C. Hay ${ }^{1}$ \\ ${ }^{1}$ MRC Centre for Regenerative Medicine, University of Edinburgh \\ Correspondence to: David C. Hay at davehay@talktalk.net
}

URL: https://www.jove.com/video/61152

DOI: doi:10.3791/61152

Keywords: Genetics, Issue 159, genome editing, CRISPR, seamless, pluripotent stem cells, piggyBac, knock-in

Date Published: 5/10/2020

Citation: Wang, Y., Smith, A.J.H., Hay, D.C. Introducing Point Mutations into Human Pluripotent Stem Cells using Seamless Genome Editing. J. Vis. Exp. (159), e61152, doi:10.3791/61152 (2020).

\section{Abstract}

Custom designed endonucleases, such as RNA-guided Clustered Regularly Interspaced Short Palindromic Repeats (CRISPR)-Cas9, enable efficient genome editing in mammalian cells. Here we describe detailed procedures to seamlessly genome edit the hepatocyte nuclear factor 4 alpha (HNF4a) locus as an example in human pluripotent stem cells. Combining a piggyBac-based donor plasmid and the CRISPR-Cas9 nickase mutant in a two-step genetic selection, we demonstrate correct and efficient targeting of the HNF4a locus.

\section{Introduction}

Human pluripotent stem cells (hPSCs) represent an unlimited source of somatic cells for research or the clinic ${ }^{1}$. Gene targeting in hPSCs offers a powerful method to study gene function during cell specification and to understand mechanisms of disease. Although efficient genome editing methods exist, modifying genes in hPSCs remains technically challenging. Standard gene targeting by homologous recombination in hPSCs occurs at low frequency or is even undetectable at some genes ${ }^{2}$, and DNA double-strand break (DSB) stimulated gene targeting (termed gene editing) is therefore necessary in these cells ${ }^{2,3}$. Additionally, transfection of hPSCs and subsequent single-cell cloning is not very efficient, even though single cell-associated apoptosis can be reduced through the use of the Rho-associated protein kinase (ROCK) inhibitor ${ }^{4}$. Finally, the potential on-target and off-target mutations at the gene of interest can also be problematic. Hence, a reliable protocol is essential to make tailored genetic changes in hPSCs.

The RNA-guided CRISPR (Clustered Regularly Interspaced Short Palindromic Repeats) and CRISPR associated protein 9 (Cas9) technology is now a well-established tool in gene editing. Transcribed CRISPR RNA (crRNA) and a trans-activating RNA (tracrRNA) form the single guide RNA (sgRNA), which complexes with Cas9 protein allowing gene specific cleavage ${ }^{5}$. The CRISPR/Cas9 system is programmable, by changing a 20-bp guide sequence of the sgRNA, meaning almost any locus that fulfils the protospacer-adjacent motif (PAM) requirement can be edited. However, the wild type Cas9 can tolerate some mismatches between its guide sequence and a DNA target, which might cause unwanted offtarget effects. To improve its specificity, a nickase mutant (Cas9n) has been developed. A Cas9n containing D10A or N863A mutation only possesses one functional nuclease domain and as a result can only nick DNA on one strand. A pair of Cas9n appropriately spaced and oriented can effectively induce DNA DSB and the off-target effects are dramatically reduced ${ }^{6}$. To ensure efficient Cas9n modification, it has been shown that two Cas9n-sgRNA should ideally be placed with a -4 to 20 bp offset and always create a $5^{5}$ overhang $^{6}$.

Cas9(n)-sgRNA induced DSB can be utilized for genome editing in hPSCs ${ }^{7,8}$. It can create gene knockout via non-homologous end joining repair, or introduce gene modification through homology-directed repair (HDR) if a donor DNA template is present. The protocol described here uses a piggyBac transposon-based donor plasmid (or targeting vector) for HDR, in which a drug resistance marker is flanked by the transposon inverted repeats. The advantage of this approach includes efficient screening and seamless gene editing as firstly demonstrated by Yusa et al. ${ }^{9,10}$. The drug selection cassette allows enrichment of cells with the integrated vector, which are subsequently screened by junction PCR to identify those derived by HDR. In addition, the drug selection cassette can be positioned to replace the target sequences for Cas9(n) cleavage, so no further DNA breakage occurs after HDR, thus eliminating 'on' target mutations resulting from Cas9(n) re-cleavage. Furthermore, by exploiting the precise excision catalyzed by piggyBac transposase, the selection marker is then excised from the genome without leaving a scar. Only the original endogenous TTAA sequence for piggyBac insertion remains after the removal of the drug selection marker ${ }^{9}$. Even if the TTAA sites have to be created by introducing substitutions, the chances of disturbing regulatory elements are reduced compared to other methods ${ }^{10}$.

Here we describe detailed procedures for implementing seamless genome editing in hPSCs. Combining a piggyBac-based donor plasmid and the CRISPR-Cas9 nickase mutant in a two-step genetic selection, we introduced two predetermined point mutations into the hepatocyte nuclear factor 4 alpha (HNF4a) gene ${ }^{11}$. This approach is reliable and efficient, and has permitted in-depth analyses of the important roles played by HNF4 $\alpha$ in endoderm and hepatocyte specification from human pluripotent stem cells ${ }^{11}$. 


\section{Designing and constructing CRISPR/Cas9n-sgRNA expression plasmids}

1. Search for 20-bp guide sequences directly upstream of any 5'-NGG near the site to be modified. A pair of single-guide RNAs (sgRNAs) is needed when the Cas9 nickase (Cas9n) from Streptococcus pyogenes is used. The pair of sgRNAs must be able to generate $5^{\prime}$ overhangs upon nicking with a -4 to 20 bp offset ${ }^{6}$.

2. Order necessary oligos and pSpCas9n-2A-puro vector.

3. Clone sgRNA into the pSpCas9n vector for co-expression with Cas9n following the published protocol ${ }^{12}$.

\section{Designing and constructing a piggyBac-based targeting vector}

1. Download the target gene sequence and search for a TTAA site near the site to be modified.

NOTE: The distance between the TTAA site and the to-be-modified site should be as small as possible. If within coding sequence and no TTAA site is present within 100-bp distance, it is necessary to introduce one by making synonymous nucleotide substitutions.

2. Design homology arms (HAs) and incorporate desired point mutations in the HAs. The optimal length of HAs should be around $500-1200$ bp. NOTE: It is recommended to introduce synonymous nucleotide substitutions to mutate the PAM sequence in order to avoid potential Cas9n re-cleavage.

3. Construct the final targeting vector following an established protocol ${ }^{10}$ NOTE: In the targeting vector used here, a PGK promoter driven puro-deltaTK selection cassette is flanked by transposon inverted repeats.

\section{Genetic editing of human pluripotent stem cells}

1. Maintain human pluripotent stem cells (hPSCs) in a humidified incubator at $37^{\circ} \mathrm{C}$ and $5 \% \mathrm{CO}_{2}$ on recombinant laminin 521 -coated surfaces $(5 \mu \mathrm{g} / \mathrm{mL})$ in mTeSR1 medium ${ }^{13}$. The cells should reach $70-85 \%$ confluency after 72 hours following a $1: 3$ split ratio.

NOTE: If present, remove spontaneously differentiated cells before daily medium change by gently aspirating them away.

2. On the day of transfection, coat enough wells of a 24 -well plate with $300 \mu \mathrm{L}$ of the laminin 521 solution $(5 \mu \mathrm{g} / \mathrm{mL})$ at $37^{\circ} \mathrm{C}$ for at least $2 \mathrm{~h}$.

3. Remove the coating solution gently and add $300 \mu \mathrm{L}$ of fresh mTeSR1 medium supplemented with $10 \mu \mathrm{M}$ ROCK inhibitor to each well, and then put the plate back to the incubator to receive cells.

NOTE: Do not allow the plates to dry at any point when the laminin coating solution is removed.

4. Move the stock hPSCs from the incubator, remove spent medium, and then wash the cells once using $1 \mathrm{~mL}$ of sterile $1 \times$ DPBS.

5. Add $1 \mathrm{~mL}$ of gentle cell dissociation reagent to each well of a 6 -well plate and incubate at $37^{\circ} \mathrm{C}$ for $6-8$ min to dissociate the cells. NOTE: Gently tap the plate and check whether the cells can detach easily to decide whether the digestion is long enough.

6. Gently pipette up and down using a P1000 tip to lift off all the hPSCs.

7. Terminate the dissociation by adding $2 \mathrm{~mL}$ of fresh $\mathrm{mTeSR} 1$ medium supplemented with $10 \mu \mathrm{M}$ ROCK inhibitor (Y-27632) to the cell suspension.

8. Mix well and transfer the single-cell suspension to a $50 \mathrm{~mL}$ tube, and centrifuge at $200 \times g$ for 3 min at room temperature.

9. Remove the supernatant, and resuspend the cells well in $2 \mathrm{~mL}$ fresh mTeSR 1 medium supplemented with $10 \mu \mathrm{M} \mathrm{ROCK}$ inhibitor.

10. Count the viable cells using a hemocytometer. Use Trypan Blue to stain and exclude dead cells.

11. Transfer $8 \times 10^{5}$ to $1 \times 10^{6}$ live cells to a $1.5 \mathrm{~mL}$ tube for each nucleofection reaction and centrifuge at $200 \times \mathrm{g}$ for $3 \mathrm{~min}$. Then carefully aspirate the supernatant.

12. Mix $3 \mu \mathrm{g}$ of the paired Cas9n-sgRNA expression plasmids and $5 \mu \mathrm{g}$ of targeting vector plasmid in $100 \mu \mathrm{L}$ of mixed nucleofection solution/ supplement from the human stem cell nucleofection kit. Use the GFP control plasmid from the kit and prepare a GFP control plasmid mix as well.

NOTE: It is important to make a maxi-prep of all plasmids to reduce endotoxin contamination before nucleofection. The concentration of the plasmids should be approximately $1 \mu \mathrm{g} / \mu \mathrm{L}$.

13. Use the DNA mix (volume $\sim 111 \mu \mathrm{L}$ ) to resuspend the prepared cells and transfer it to an electroporation cuvette (provided with the nucleofection kit), avoiding any bubbles.

14. Electroporate the cells using the nucleofection device by selecting the optimized conditions for human pluripotent stem cells. NOTE: If using the nucleofection kit for human pluripotent stem cells for the first time, it is necessary to test the electroporation program and choose the most efficient one.

15. Immediately add $500 \mu \mathrm{L}$ of fresh and warm to $37^{\circ} \mathrm{C}$ mTeSR 1 medium supplemented with $10 \mu \mathrm{M}$ ROCK inhibitor to the electroporated cells. Transfer the mix to 2 wells of a 24-well plate prepared from steps 3.2 and 3.3.

16. Quickly put the plate back to the $37^{\circ} \mathrm{C} / 5 \% \mathrm{CO}_{2}$ incubator and allow the cells to recover.

17. 12-16 $\mathrm{h}$ later, change cell maintenance medium. If the cells established cell-cell contact, withdraw the ROCK inhibitor, if not, continue supplementing the medium with the inhibitor.

18. Between $24-48 \mathrm{~h}$, check the nucleofection efficiency by examining GFP expression in the control cells. GFP positive cells should be at least 30 percent

19. $48 \mathrm{~h}$ after nucleofection, start selecting cells by supplementing the $\mathrm{mTeSR} 1$ medium with $1 \mu \mathrm{g} / \mathrm{mL}$ puromycin.

20. $72 \mathrm{~h}$ after nucleofection, supplement the mTeSR1 medium with $0.5 \mu \mathrm{g} / \mathrm{mL}$ puromycin. If the cell confluency is lower than $30 \%$, also supplement the medium with $10 \mu \mathrm{M}$ ROCK inhibitor.

21. 4-6 days post-nucleofection, passage the puromycin-resistant cells to $10-15 \times 96$-well plates at the concentration of 0.8 cells/well. NOTE: It is essential to supplement the medium with $10 \mu \mathrm{M} \mathrm{ROCK}$ inhibitor and $0.5 \mu \mathrm{g} / \mathrm{mL}$ puromycin.

22. Maintain those cells at $37^{\circ} \mathrm{C} / 10 \% \mathrm{CO}_{2}$ for $10-12$ days to form single cell-derived colonies. Top up medium once 7 days after seeding NOTE: Increased $\mathrm{CO}_{2}$ level helps single hPSCs to form colonies from our experience. 
23. Mark wells containing a single colony and replace the medium with fresh mTeSR1 medium containing $0.5 \mu \mathrm{g} / \mathrm{mL}$ puromycin but no ROCK inhibitor.

NOTE: From this point on, the cells will be grown under $37^{\circ} \mathrm{C} / 5 \% \mathrm{CO}_{2}$.

24. Two days later, change medium for wells containing undifferentiated colonies. Supplement the medium with $10 \mu \mathrm{M}$ ROCK inhibitor and 0.5 $\mu \mathrm{g} / \mathrm{mL}$ puromycin.

NOTE: In some wells, the cells might have differentiated and need to be discarded.

25. Use P2 pipette tips to scrape off the colonies gently. Transfer the cell suspension derived from one colony into 2 new wells on separate $96-$ well plates, and use one for genotyping and one for maintaining.

NOTE: It is important to carefully maintain the colonies and keep the spontaneous differentiation level to the minimum.

26. Take the plate containing cells for genotyping out once the confluency in most wells reached $50 \%$ or above. Dump the spent medium and then wash the cells once with DPBS.

27. Lyse the cells in well using Bradley lysis buffer and isolate genomic DNA from each well in the plate following associated protocol (https:// mcmanuslab.ucsf.edu/protocol/dna-isolation-es-cells-96-well-plate).

28. Use a three-primer junction PCR method to genotype both left and right homology arms independently ${ }^{10}$. NOTE: A scheme showing the PCR method is presented in Figure 2.

29. Send the junction PCR products for both homology arms from 4-5 colonies for Sanger sequencing and get the sequences. NOTE: The sequencing result of 2 established colonies is shown in Figure 3A.

30. Keep colonies with the correct genotype and discard the rest.

31. Expand the correct colonies under continuous puromycin selection and freeze them at the earliest possible passage.

\section{Removing transposon from targeted human pluripotent stem cells}

1. Maintain one colony with correct genotype under $0.5 \mu \mathrm{g} / \mathrm{mL}$ puromycin selection.

2. Nucleofect $8 \times 10^{5}$ to $1 \times 10^{6}$ cells with $5 \mu$ g hyperactive transposase (pCMV-hyPBase) as described above (Section 3, steps $3.4-3.18$ ). Perform a GFP control nucleofection in parallel. NOTE: Puromycin should be removed from the mTeSR1 medium immediately after nucleofecting these cells.

3. Grow and screen for cells with the puro-deltaTK selection cassette removed following published procedures ${ }^{10}$ NOTE: It is important to follow the original procedures carefully. FIAU, or 1-(2-deoxy-2-fluoro- $\beta$-d-arabinofuranosyl)-5-iodouracil), was used as a thymidine analog for Herpes simplex virus-derived thymidine kinase (HSV-tk)-based negative selection.

4. Sequence the modified region to confirm the removal of the transposon. NOTE: The sequencing result of 3 established colonies is shown in Figure 3B.

5. Keep colonies with the correct genotype and expand for further usage.

6. Perform characterization of pluripotency markers in the chosen colonies before using them for further analysis. NOTE: The characterization of two established colonies is shown in Figure 4.

\section{Representative Results}

Targeting vector-based knock-in strategy

The hepatocyte nuclear factor 4 alpha $(\mathrm{HNF} 4 \alpha)$ gene was chosen for targeted genome editing to introduce two point mutations into exon 8 . A pair of Cas9n-sgRNA with $11 \mathrm{bp}$ offset was designed close to the site to be modified. The piggyBac-based targeting vector worked as the homology-directed repair template for introducing the desired point mutations. A 967 bp 5'-HA and a 1142 bp 3'-HA incorporating synonymous nucleotide substitutions or the desired point mutations were amplified and cloned into the final targeting vector. The piggyBac insertion site was $16 \mathrm{bp}$ and $22 \mathrm{bp}$ away from the two desired point mutations. Colonies containing the puro-deltaTK selection cassette were selected with puromycin in the first round. Once the selection cassette was removed by transposase excision in the second round, the targeted site was modified seamlessly with only the desired point mutations incorporated in the gene (Figure 1).

Genetic editing in human pluripotent stem cells

To screen for correctly targeted cells, a three primer-based PCR method was used for genotyping (Figure 2). Sanger sequencing was performed to confirm the PCR results (Figure 3A). Post removal of the selection cassette, the modified region was sequenced again to confirm the correct introduction of desired point mutations (Figure 3B).

Colony establishment and characterization of edited human pluripotent stem cells Colonies with the correct genotype were selected and expanded as needed. The established colonies need to be characterized before being used for further analysis. The edited cells possess the same morphology as the parental cells (Figure 4A). They also express representative human pluripotent stem cell markers, including transcription factors NANOG and OCT4 (Figure 4B), as well as cell surface markers SSEA4 and TRA-1-60 (Figure 4C). 


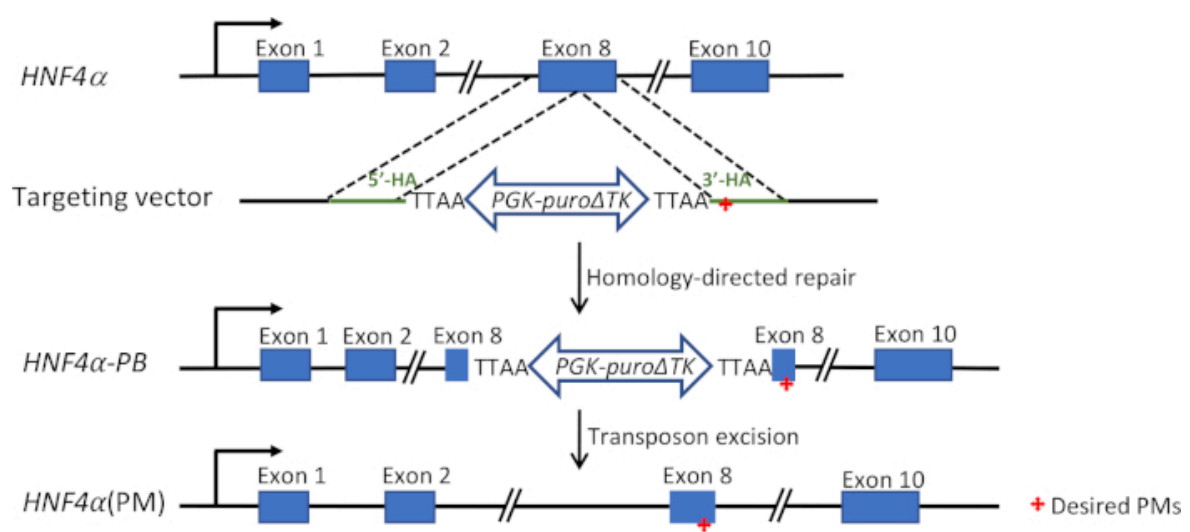

Figure 1: Targeting vector-based knock-in strategy ${ }^{11}$. A pair of Cas9n-sgRNA expression plasmids were used to induce DNA double-strand break in exon 8 of the HNF4a gene. A targeting vector with a selection cassette was used to introduce predetermined point mutations located $16 \mathrm{bp}$ and $22 \mathrm{bp}$ away. This selection cassette was contained within the piggyBac transposon, consisting of a positive-negative selection marker (puro-deltaTK) driven by a constitutively active promoter (PGK). Via homology-directed repair pathway, the targeted cells incorporated the selection cassette. Transposon excision mediated by transposase results in a seamless modification with only the point mutations present. Red cross indicates the location of desired point mutations. $\mathrm{HA}=$ homology arm; $\mathrm{PB}=$ piggyBac; $\mathrm{PM}=$ point mutation. Please click here to view a larger version of this figure.

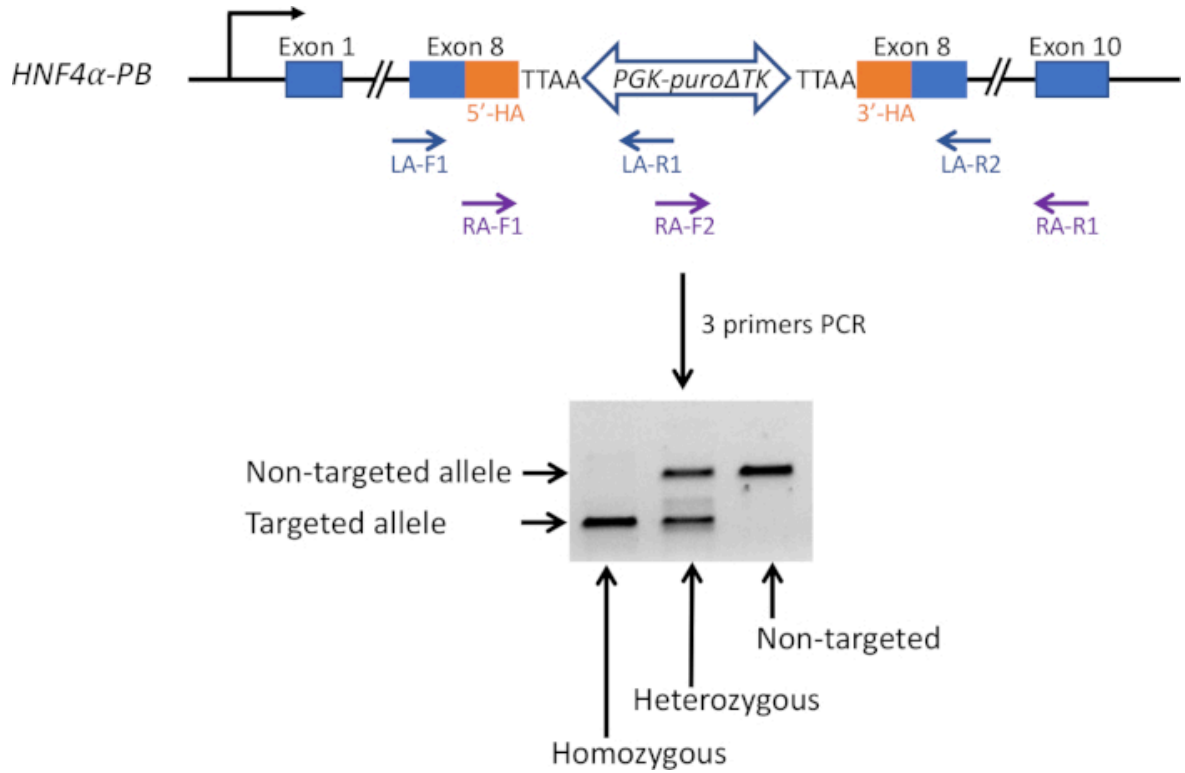

Figure 2: Three primer-based PCR method. To screen gene targeted cells, PCR-based genotyping was used. Three primers, LA-F1, -R1 and -R2 were used to amplify the left homology arm region. Independently, RA-F1, -F2 and -R1 were used to amplify the right homology arm region. Based on the gel electrophoresis result, non-targeted cells, and heterozygous and homozygous cells, were distinguished from each other. Please click here to view a larger version of this figure. 
A

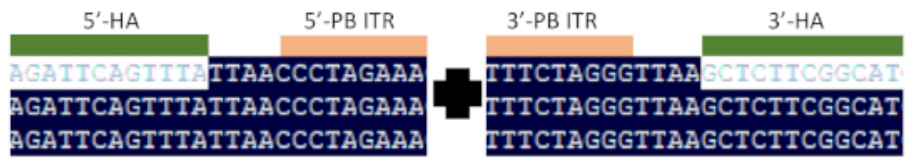

B

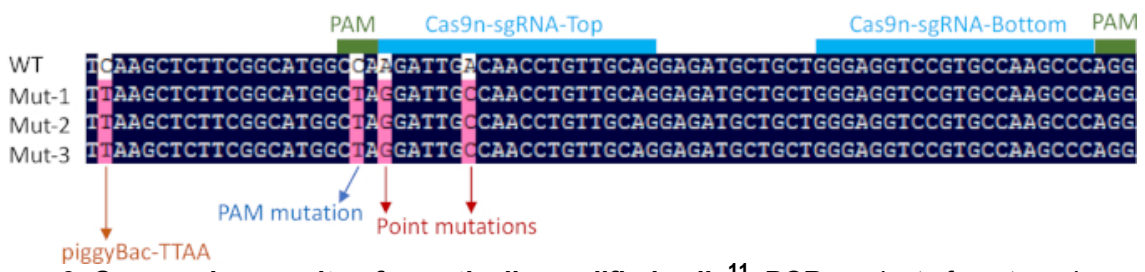

Figure 3: Sequencing results of genetically modified cells $\mathbf{s}^{11}$. PCR products from two clones were sequenced and confirmed the correct insertion of the selection cassette at the targeted locus (A). 5' and 3' piggyBac inverted terminal repeats (ITR) were flanked by the TTAA direct repeats. Three clones were sequenced post transposon excision (B). A pair of Cas9n-sgRNA with 11 bp offset was used to introduce DNA double-strand break. Two predetermined point mutations ( $A$ to $G$ and $A$ to $C$ ) were introduced into the gene. One synonymous mutation was introduced to mutate the protospacer-adjacent motif (PAM), and another one to create the TTAA site necessary for piggyBac excision. Please click here to view a larger version of this figure.
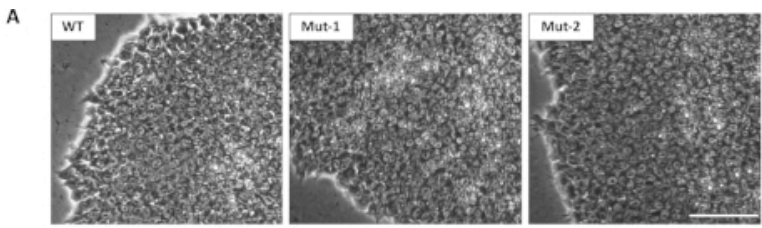

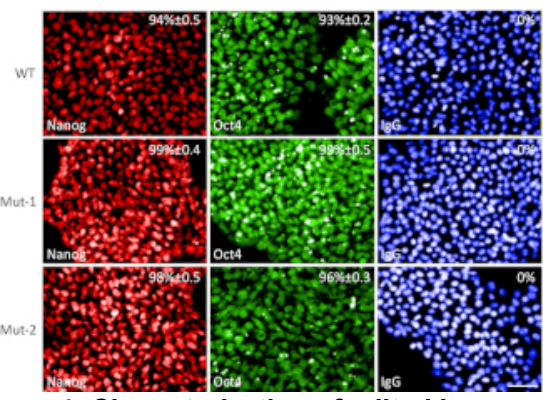

C

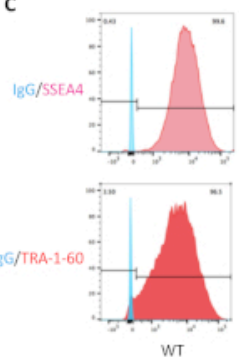

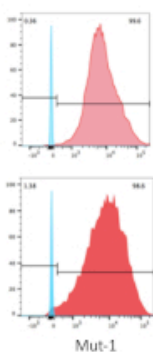

Mut-1

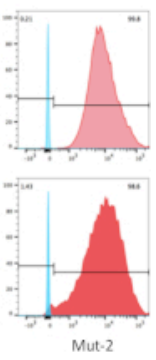

Figure 4: Characterization of edited human pluripotent stem cells. Morphology of two edited cell lines and the parental cells (A), scale bar $=100 \mu \mathrm{m}$. The expression of pluripotent stem cell markers NANOG and OCT4 examined by immunostaining $(\mathrm{B}, \mathrm{scale}$ bar $=50 \mu \mathrm{m})$, in addition to SSEA4 and TRA-1-60 by flow cytometry (C) in two modified cell lines and the parental cells. IgG was used as a negative control. DAPI was used to stain the nucleus. The percentages were calculated as the average of three independent experiments. Please click here to view a larger version of this figure.

\section{Discussion}

The protocol described herein can be used to introduce predetermined point mutations into an endogenous locus in hPSCs. The combination of a piggyBac-based targeting vector and paired CRISPR/Cas9n expression plasmids proved to be reliable and efficient ${ }^{11}$. Following HNF4a gene editing, 12 out of 43 analyzed clones were correctly targeted as determined by junction PCR screening. Specifically, the biallelic targeting efficiency was about $21 \%(9 / 43)$ and the monoallelic targeting efficiency was around $7 \%(3 / 43)$.

Following targeting experiments, it is crucial to maintain puromycin selection to keep the targeted locus accessible to the piggyBac transposase during the first round of screening. This will minimize the silencing of the PGK promoter-driven puro-deltaTK selection cassette and reduce the background in the second round of screening ${ }^{10}$. A recent report showed that CAG promoter is more resistant to silencing than PGK promoter in $\mathrm{hPSCs}^{14}$. Changing the promoter for the selection cassette could therefore improve this system in the future. It is also important to compare the number of resistant colonies from hyPBase- and GFP-transfected cells. Upon successful removal of the transposon, there should be more surviving colonies from the hyPBase- than GFP-transfected cells. In addition to PCR analysis of transposon excision, direct sequencing of the modified region is required to confirm the excision fidelity.

Based on Yusa's piggyBac transposon-based targeting vector strategy ${ }^{9,10}$, the procedures detailed above focused on improving hPSCs nucleofection and single cell cloning efficiency. Cell seeding density was optimized to reduce the likelihood of heterogeneous colony formation. We also employed 10-12 days culture under $10 \% \mathrm{CO}_{2}$ to enhance colony production for genotype screening. In our experience, around 20 colonies could be obtained from each 96 -well plate. Although this step might take more time than other methods, it is reliable and highly efficient. 
Based on the need, targeting vectors can be designed to introduce or correct point mutations, to create reporter cell lines, as well as knockout gene expression. In conclusion, the combination of the CRISPR/Cas9(n) system and a targeting vector is an efficient way to deliver different types of genetically modified hPSCs.

\section{Disclosures}

D.C.H is a co-founder, shareholder, and director of Stemnovate Limited.

\section{Acknowledgments}

We thank Kosuke Yusa for sharing the pMCS-AAT_PB-PGKpuroTK and pCMV-hyPBase plasmids. We thank Jia-Yin Yang and Karamjit SinghDolt for helpful discussions on genome editing. D.C.H. lab is supported by an award from the Chief Scientist Office (TC/16/37) and the UK Regenerative Medicine Platform (MR/L022974/1). Y.W. was supported by a PhD scholarship funded by the Chinese Scholarship Council and the University of Edinburgh.

\section{References}

1. Rashidi, H. et al. 3D human liver tissue from pluripotent stem cells displays stable phenotype in vitro and supports compromised liver function in vivo. Archives of Toxicology. 92 (10), 3117-3129 (2018).

2. Hockemeyer, D. et al. Efficient targeting of expressed and silent genes in human ESCs and iPSCs using zinc-finger nucleases. Nature Biotechnology. 27 (9), 851-857 (2009).

3. Porteus, M.H., Baltimore, D. Chimeric nucleases stimulate gene targeting in human cells. Science. 300 (5620), 763 (2003).

4. Watanabe, K. et al. A ROCK inhibitor permits survival of dissociated human embryonic stem cells. Nature Biotechnology. 25 (6), 681-686 (2007).

5. Jinek, M. et al. A programmable dual-RNA-guided DNA endonuclease in adaptive bacterial immunity. Science. 337, 816-822 (2012).

6. Ran, F.A. et al. Double nicking by RNA-guided CRISPR cas9 for enhanced genome editing specificity. Cell. 154 (6), $1380-1389$ (2013).

7. Cong, L. et al. Multiplex genome engineering using CRISPR/Cas systems. Science. 339 (6121), 819-824 (2013).

8. Mali, P. et al. RNA-guided human genome engineering via Cas9. Science. 339 (6121), 823-826 (2013).

9. Yusa, K. et al. Targeted gene correction of a1-antitrypsin deficiency in induced pluripotent stem cells. Nature. 478 (7369), $391-394$ (2011).

10. Yusa, K. Seamless genome editing in human pluripotent stem cells using custom endonuclease-based gene targeting and the piggyBac transposon. Nature Protocols. 8 (10), 2061-2078 (2013).

11. Wang, Y. et al. Multiomics Analyses of HNF4a Protein Domain Function during Human Pluripotent Stem Cell Differentiation. iScience. 16, 206-217 (2019).

12. Ran, F.A., Hsu, P.D., Wright, J., Agarwala, V., Scott, D.A., Zhang, F. Genome engineering using the CRISPR-Cas9 system. Nature Protocols. 8 (11), 2281-2308 (2013).

13. Wang, Y. et al. Defined and Scalable Generation of Hepatocyte-like Cells from Human Pluripotent Stem Cells. Journal of Visualized Experiments. (121), 1-8 (2017).

14. Eggenschwiler, R. et al. Improved bi-allelic modification of a transcriptionally silent locus in patient-derived iPSC by Cas 9 nickase. Scientific Reports. 6, 1-14 (2016). 